FERMILAB-TM-2322-AD-E

CDF/DOC/TRIGGER/CDFR/7621

Version 1.0 - May 2, 2005

\title{
Influence of Luminosity Leveling on the CDF-II B-Physics Program
}

\author{
Matthew Jones \\ Purdue University \\ Jonathan Lewis
}

Fermilab

\section{Introduction}

The effective bandwidth of the CDF-II level 1 trigger is approximately $25 \mathrm{kHz}$. Some of this bandwidth is used to record events that form the data sets used by the high $p_{T}$ physics analyses. The remaining bandwidth is used by triggers that are sensitive to hadronic $B$ decays and provide one of the most important samples used for the study of $B_{s}^{0}$ mixing. At high luminosities, the hadronic $B$ triggers have rates that greatly exceed the available bandwidth. Rather than incur large dead-times associated with these excessive rates, these $B$ triggers are prescaled to limit the total trigger rate to the effective level 1 trigger bandwidth. The prescales are dynamically adjusted as the store progresses so that all of the bandwidth that is not used for the high $p_{T}$ physics program is used to record hadronic $B$ triggers.

In principle, the luminosity could be held at a more constant level throughout the store in such a way that the integrated luminosity would be the same as that obtained from a normal store. It has been suggested that this would allow $B$ triggers to be recorded with lower prescales and consequently with higher $B_{s}^{0}$ signal efficiencies. This note describes a parametric model of the high $p_{T}$ and hadronic $B$ triggers used by $\mathrm{CDF}$ and compares the yields of reconstructed $B_{s}^{0}$ decays that would result with and without luminosity leveling. 


\begin{tabular}{|l||c|c|c|c|c|l|}
\hline parameter & 2005 & 2006 & 2007 & 2008 & 2009 & units \\
\hline \hline Initial $\mathcal{L}$ & 77 & 96 & 219 & 284 & 284 & $\mathrm{ub}^{-1} \mathrm{~s}^{-1}$ \\
Store length & 25 & 20 & 20 & 15 & 15 & hours \\
Store hours per week & 100 & 100 & 100 & 100 & 100 & hours \\
Weeks of operations per year & 42 & 42 & 42 & 42 & 42 & weeks \\
Integrated $\mathcal{L}$ per week & 12 & 17 & 38 & 50 & 50 & $\mathrm{pb}^{-1}$ \\
\hline
\end{tabular}

Table 1: Tevatron operating parameters used for $B_{s}^{0}$ yield projections.

\section{Parametric Model}

We quantify the possible benefits of luminosity leveling in terms of the number of reconstructed $B_{s}^{0}$ decays that could be recorded compared with the number that would be reconstructed with current luminosity profiles. The model used to calculate these yields uses as input

- the projected Tevatron operating parameters in future years

- the luminosity profile for a single store

- the effective level 1 trigger bandwidth

- a parameterization of the high $p_{T}$ trigger rate as a function of instantaneous luminosity

- a parameterization of the hadronic $B$ trigger rates as functions of instantaneous luminosity

- the $B_{s}^{0}$ signal cross section which is independent of instantaneous luminosity.

The following sections describe in detail the assumptions that have gone into modeling these effects and their justification.

\subsection{Tevatron operating parameters}

Future upgrades to the accelerator complex primarily result in an increased $\bar{p}$ stacking rate which translates into increased Tevatron luminosity in two ways. First, more anti-protons will be available for each store and second, new stores will be shot more frequently due to the shorter time needed to accumulate sufficient anti-protons to shoot again. Table 1 summarizes the expected parameters that will influence the integrated luminosity over the years 2005-2009[1]. 


\subsection{Luminosity Profile}

The instantaneous luminosity as a function of time throughout a typical store is parameterized by the function

$$
\mathcal{L}(t)=\mathcal{L}_{0}\left(f e^{-t / \tau_{1}}+(1-f) e^{-t / \tau_{2}}\right)
$$

where $\mathcal{L}_{0}$ is the initial luminosity, obtained from Table 1 , while $f=0.38, \tau_{1}=$ 3.8 hours and $\tau_{2}=23.0$ hours are parameters that have been determined from the luminosity profile of past Tevatron stores.

The luminosity integrated over a store of duration $T$ is then

$$
\int_{0}^{T} \mathcal{L} d t=\mathcal{L}_{0}\left(\tau_{1} f\left(1-e^{-T / \tau_{1}}\right)+\tau_{2}(1-f)\left(1-e^{-T / \tau_{2}}\right)\right)
$$

from which the integrated luminosity per week is calculated, in conjunction with the store duration and the number of store hours per week. This calculation yields weekly integrated luminosities that are in good agreement with those listed in Table 1: 12.1, 16.8 and $38.3 \mathrm{pb}^{-1}$ in years $2005-2007$ and $56.3 \mathrm{pb}^{-1}$ in 2008 and 2009 .

In the study of luminosity leveling, we assume the most optimistic scenario possible, whereby the instantaneous luminosity is held constant at a value $\overline{\mathcal{L}}=\left(\int \mathcal{L} d t\right) / T$ which yields the same luminosity integrated per store of the same duration. In practice, the integrated luminosity with luminosity leveling would probably be lower than this and operation of the Tevatron in this way may also have a negative impact on the quality of the data recorded by CDF. Nevertheless, we use only this simple model to place an upper limit on the $B_{s}^{0}$ yield obtained with luminosity leveling.

\subsection{High $p_{T}$ Trigger Rate}

We have considered three possible scenarios to estimate the level 1 trigger bandwidth that will be devoted to the study of high $p_{T}$ processes as follows:

- Jet and electromagnetic triggers only

- As above but also including muon and di-electron triggers

- As above but also including di-tau triggers.

The effective trigger cross sections for these three scenarios and the resulting level 1 trigger rates are shown in Figure 1. These cross sections were determined from parameterizations made by XMON and take into account the overlap between jet and electromagnetic triggers. The second scenario provides triggers used for the SUSY di-lepton program. The remaining level 1 triggers either contribute little to the total rate, overlap with the high $p_{T}$ scenarios studied here, or are of the class of high rate track triggers that is the subject of this note. 

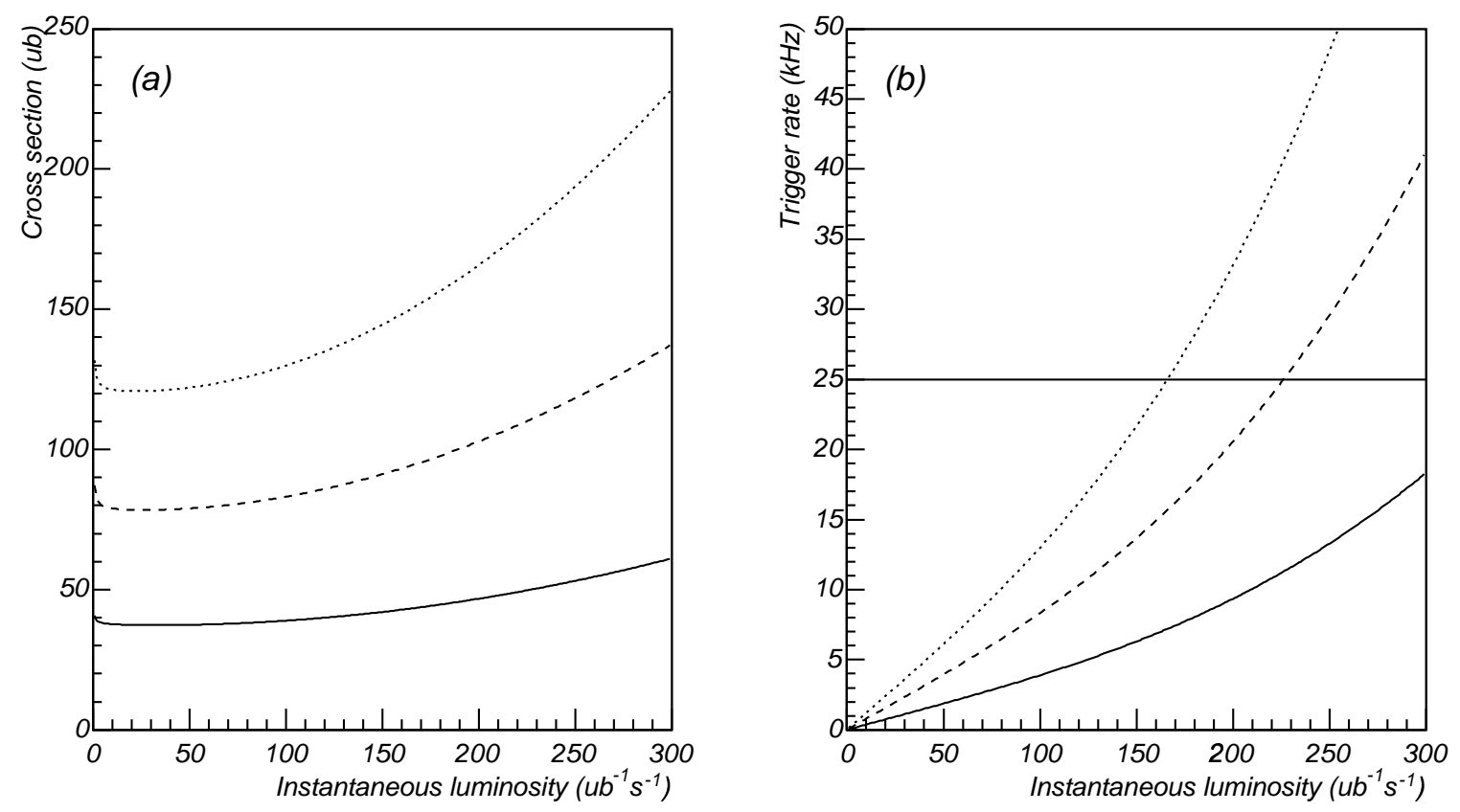

Figure 1: Effective trigger cross sections (a) and rates (b) for the three high $p_{T}$ trigger scenarios. The effective level 1 trigger bandwidth is indicated by the horizontal line at $25 \mathrm{kHz}$. 


\subsection{Hadronic $B$-Trigger Rates}

There are three level 1 triggers that are used to select events that may contain hadronic $B$ decays. All three require the presence of two XFT tracks but impose the following additional requirements:

- Scenario C

- Both tracks must have $p_{T}>2.5 \mathrm{GeV} / c$

- Opposite charge

$-\sum p_{T}>6.5 \mathrm{GeV} / c$

$-2^{\circ}<\Delta \phi<135^{\circ}$

- Scenario A

- Both tracks must have $p_{T}>2.0 \mathrm{GeV} / c$

$-\sum p_{T}>5.5 \mathrm{GeV} / c$

- Opposite charge

$-2^{\circ}<\Delta \phi<135^{\circ}$

- Scenario Low

- Both tracks must have $p_{T}>2.0 \mathrm{GeV} / c$

$-2^{\circ} \Delta \phi<135^{\circ}$

In this study, we assume that additional criteria are imposed on all three triggers as follows:

- Confirmation of XFT tracks by stereo segments

- $\Delta \phi<90^{\circ}$

- $m_{T}<4 \mathrm{GeV} / c^{2}$

The cross sections are estimated by applying these trigger criteria to data recorded with highly prescaled backup triggers. The extrapolation to high luminosities is performed by analyzing the cross sections as functions of the number of reconstructed primary vertices[2] and are shown in Figure 2. The benefits of the upgraded XFT trigger were estimated by matching XFT tracks to stereo segments found in superlayers 3 and 7 . This gave results similar to those described in previous studies[3] but would benefit from a more realistic simulation of the proposed hardware upgrades. 


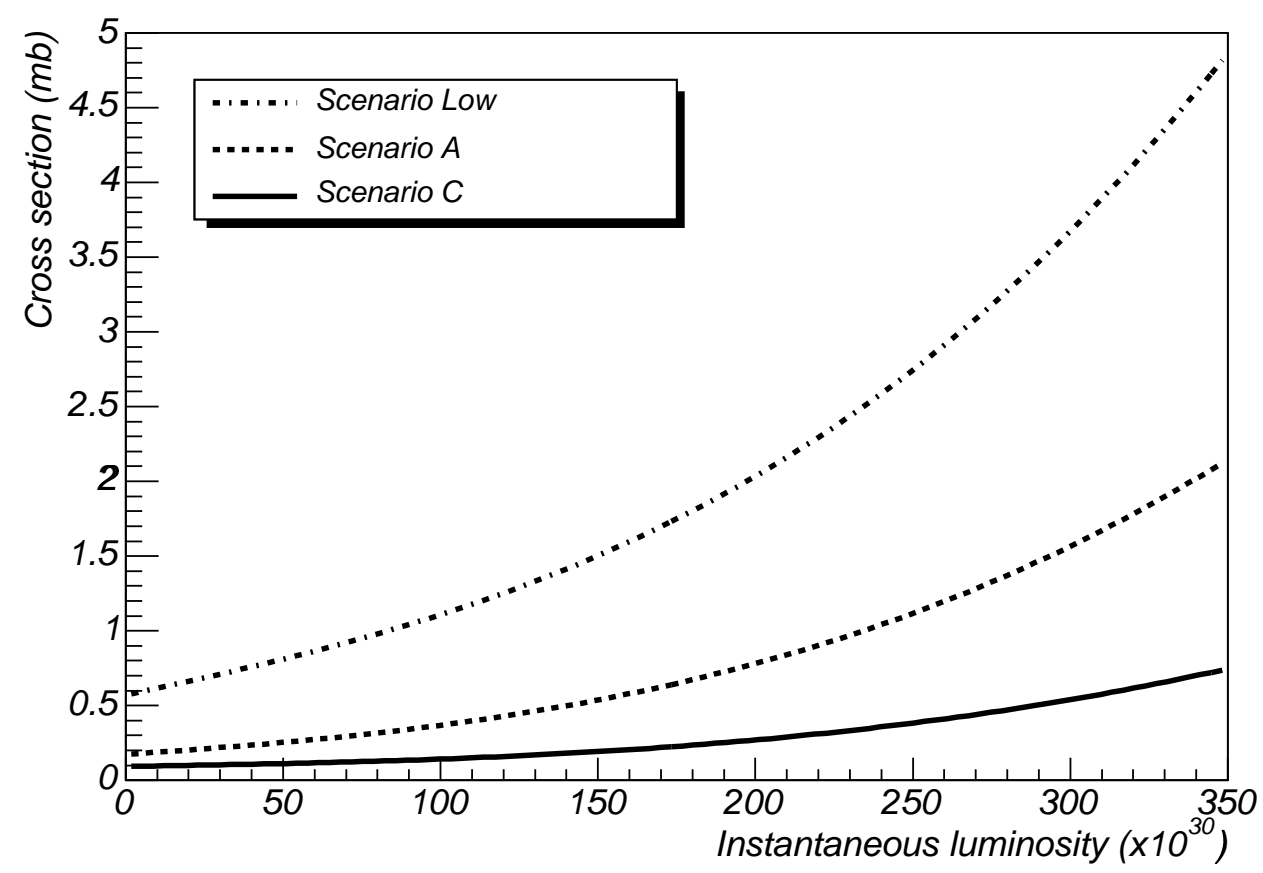

Figure 2: Un-prescaled cross section for the Scenario C, Scenario A, and Scenario Low triggers with the additional requirements described in Section 2.4. 


\section{5 $\quad B_{s}^{0}$ Signal Yields}

The only fully reconstructed $B_{s}^{0}$ decay mode considered for this analysis is $B_{s}^{0} \rightarrow$ $D_{s}^{-} \pi^{+}$. The yield of $B_{s}^{0}$ decays reconstructed in this way has been studied using approximately $65 \mathrm{pb}^{-1}$ of data recorded using the Scenario $\mathrm{C}$ and Scenario A hadronic triggers[4]. The cross section obtained from the higher threshold Scenario $\mathrm{C}$ trigger was measured to be $1.080 \mathrm{pb}$, while the cross section obtained from events that satisfied the Scenario A, but not the Scenario C trigger, was determined to be $0.713 \mathrm{pb}$. We make the naive assumption that the cross section for reconstructing $B_{s}^{0}$ decays in events recorded with the Scenario Low trigger, but not Scenarios A or C, will be half this value, or $0.357 \mathrm{pb}$. In practice, very few events will be recorded with the Scenario Low trigger, so this assumption has only a small effect on the comparisons presented here. These measurements have a relative uncertainty of approximately $20 \%$ due to the limited statistics available at the time the study was performed. Although we can now make more precise estimates of the signal cross section, it would only change the overall scale of the $B_{s}^{0}$ yield and would not affect the comparison of the relative numbers of $B_{s}^{0}$ decays recorded with or without luminosity leveling.

The effective cross sections, from which the reconstructed $B_{s}^{0}$ yield is determined are these cross sections divided by the instantaneous prescale factor that is needed to maintain a total level 1 trigger rate that is less than the available bandwidth. The instantaneous rate at which reconstructible $B_{s}^{0}$ decays are recorded from trigger scenario $i$, is then

$$
r_{i}(t)=\mathcal{L}(t) \frac{\sigma_{i}}{p_{i}(t)}
$$

and the total number of $B_{s}^{0}$ decays recorded in a store of duration $T$ is

$$
N_{i}=\int_{0}^{T} \mathcal{L}(t) \frac{\sigma_{i}}{p_{i}(t)} d t
$$

This integral is evaluated numerically to estimate the total yield in one store, and the yearly yield is obtained by multiplying the result by the number of stores per week and the number of weeks of operation per year, for each year listed in Table 1.

\section{Results}

The elements of the model just described are used to estimate the yields of reconstructed $B_{s}^{0}$ decays as follows. For a given luminosity profile, the luminosity dependent high $p_{T}$ trigger cross section, $\sigma_{0}(\mathcal{L})$, is calculated for one of the scenarios described in section 2.3. From this cross section, the rate, $R_{0}(\mathcal{L})=\mathcal{L} \sigma_{0}(\mathcal{L})$, of high $p_{T}$ triggers is calculated, but it is set to the maximum effective level 1 bandwidth, $B_{0}=25 \mathrm{kHz}$, if this un-prescaled rate exceeds this value. The remaining bandwidth, $B(\mathcal{L})=B_{0}-R_{0}(\mathcal{L})$, can be used for hadronic $B$ triggers. 


\begin{tabular}{|c||c|c|c|}
\hline Year & optimistic & nominal & pessimistic \\
\hline \hline 2005 & 976.1 & 958.9 & 938.4 \\
2006 & 1242.8 & 1205.9 & 1163.3 \\
2007 & 1648.3 & 1359.8 & 1046.8 \\
2008 & 1459.3 & 982.7 & 545.8 \\
2009 & 1459.3 & 982.7 & 545.8 \\
\hline Total & 6785.8 & 5490.0 & 4240.0 \\
\hline
\end{tabular}

Table 2: $B_{s}^{0}$ signal yields by year for the optimistic, nominal and pessimistic high $p_{T}$ trigger scenarios obtained from stores with the normal luminosity profile.

The level 1 Scenario $\mathrm{C}$ hadronic trigger cross section, $\sigma_{C}(\mathcal{L})$, is calculated using the parameterization described in section 2.4. The prescale factor, $p_{C}$, that is required to limit the trigger rate to the available bandwidth is calculated from $p_{C}=\mathcal{L} \sigma_{C}(\mathcal{L}) / B(\mathcal{L})$. The rate at which reconstructible $B_{s}^{0}$ meson decays are recorded and the total number recorded during the store are calculated using Equations 3 and 4. If the high $p_{T}$ and Scenario $\mathrm{C}$ triggers to not completely saturate the available level 1 trigger bandwidth, then the same procedure is used to determine the prescales and yields for the Scenario A and Scenario Low triggers.

\subsection{Yields from Normal Stores}

The cross sections, rates, prescale factors, signal rates and signal yields were calculated using the luminosity profile parameterized by Equation 1 using the parameters for each of the years listed in Table 1. Figures 3 and 4 show these quantities as a function of time throughout one store for the years 2006 and 2008, respectively, using the nominal high $p_{T}$ trigger cross section parameterization. Table 2 lists the yearly yields of reconstructed $B_{s}^{0}$ mesons for the three high $p_{T}$ trigger scenarios.

\subsection{Yields from Luminosity Leveled Stores}

Figures 5 and 6 show the trigger rates, prescales and cross sections for stores in which the luminosity is held fixed at a value $\overline{\mathcal{L}}$ such that the integrated luminosity of the store is the same as one of the same duration but with the normal luminosity profile. In this case, since the luminosity is constant, all trigger cross sections, prescales and rates are also constant.

The yearly yields of reconstructed $B_{s}^{0}$ mesons obtained from luminosity leveled stores are listed in Table 3. The relative yields, compared with what would have been obtained with a normal luminosity profile, are shown in Table 4. 

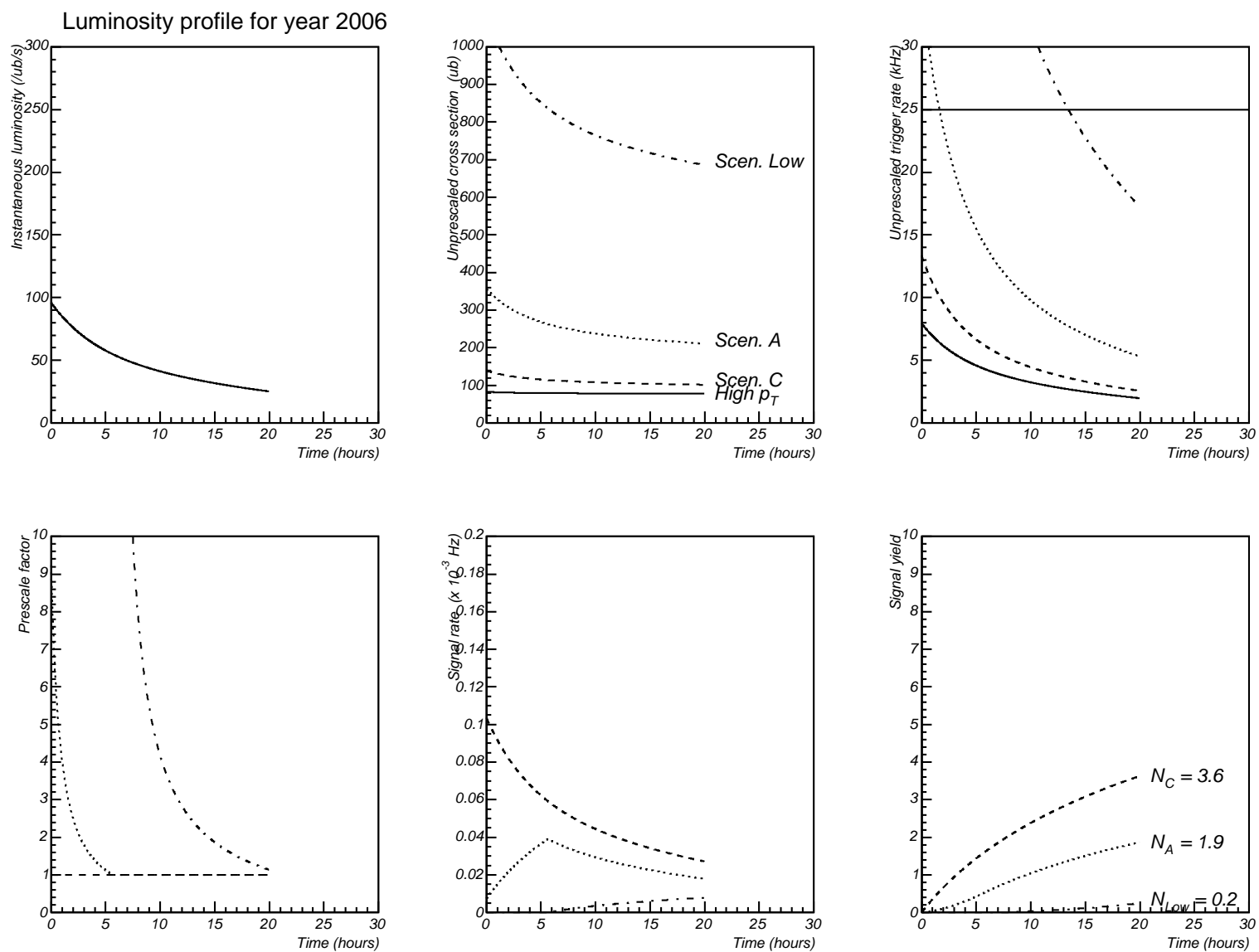

Figure 3: Instantaneous luminosity, cross sections, trigger rates prescales, $B_{s}^{0}$ signal rates and signal yields for the 2006 luminosity with the nominal high $p_{T}$ trigger scenario.

\begin{tabular}{|c||c|c|c|}
\hline Year & optimistic & nominal & pessimistic \\
\hline \hline 2005 & 1003.9 & 993.6 & 983.0 \\
2006 & 1309.5 & 1296.4 & 1282.7 \\
2007 & 1881.6 & 1748.8 & 1221.2 \\
2008 & 1497.9 & 862.7 & 169.5 \\
2009 & 1497.9 & 862.7 & 169.5 \\
\hline Total & 7190.8 & 5764.1 & 3825.9 \\
\hline
\end{tabular}

Table 3: $B_{s}^{0}$ signal yields by year for the optimistic, nominal and pessimistic high $p_{T}$ trigger scenarios obtained from stores with a constant luminosity. 

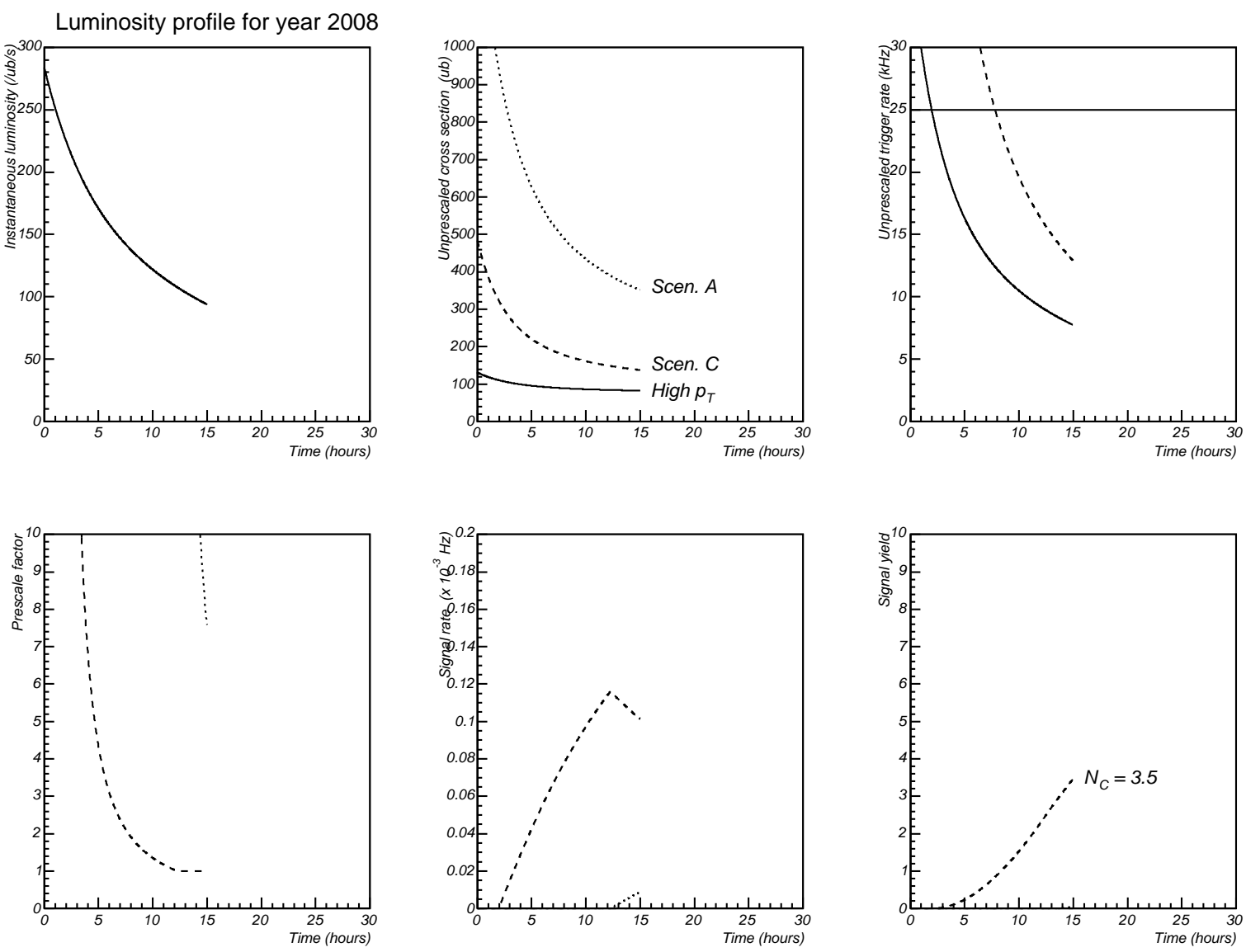

Figure 4: Instantaneous luminosity, cross sections, trigger rates prescales, $B_{s}^{0}$ signal rates and signal yields for the 2008 luminosity with the nominal high $p_{T}$ trigger scenario.

\begin{tabular}{|c||c|c|c|}
\hline Year & optimistic & nominal & pessimistic \\
\hline \hline 2005 & 1.028 & 1.036 & 1.000 \\
2006 & 1.054 & 1.075 & 1.103 \\
2007 & 1.142 & 1.286 & 1.167 \\
2008 & 1.026 & 0.878 & 0.311 \\
2009 & 1.026 & 0.878 & 0.311 \\
\hline
\end{tabular}

Table 4: Relative $B_{s}^{0}$ signal yields for luminosity leveled stores compared with normal stores for the optimistic, nominal and pessimistic high $p_{T}$ trigger scenarios. 

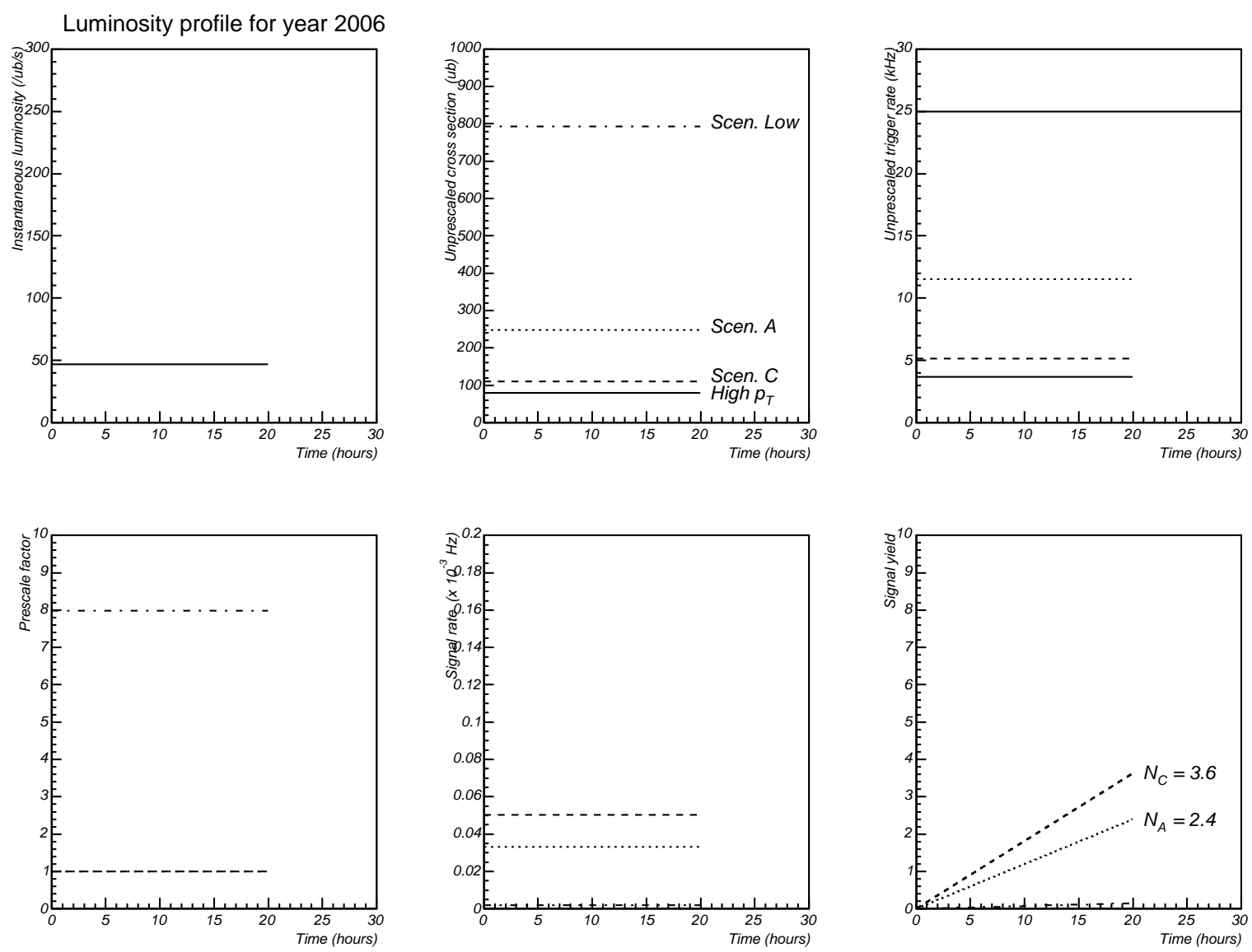

Figure 5: Instantaneous luminosity, cross sections, trigger rates prescales, $B_{s}^{0}$ signal rates and signal yields for a luminosity leveled store in 2006 with the nominal high $p_{T}$ trigger scenario. 

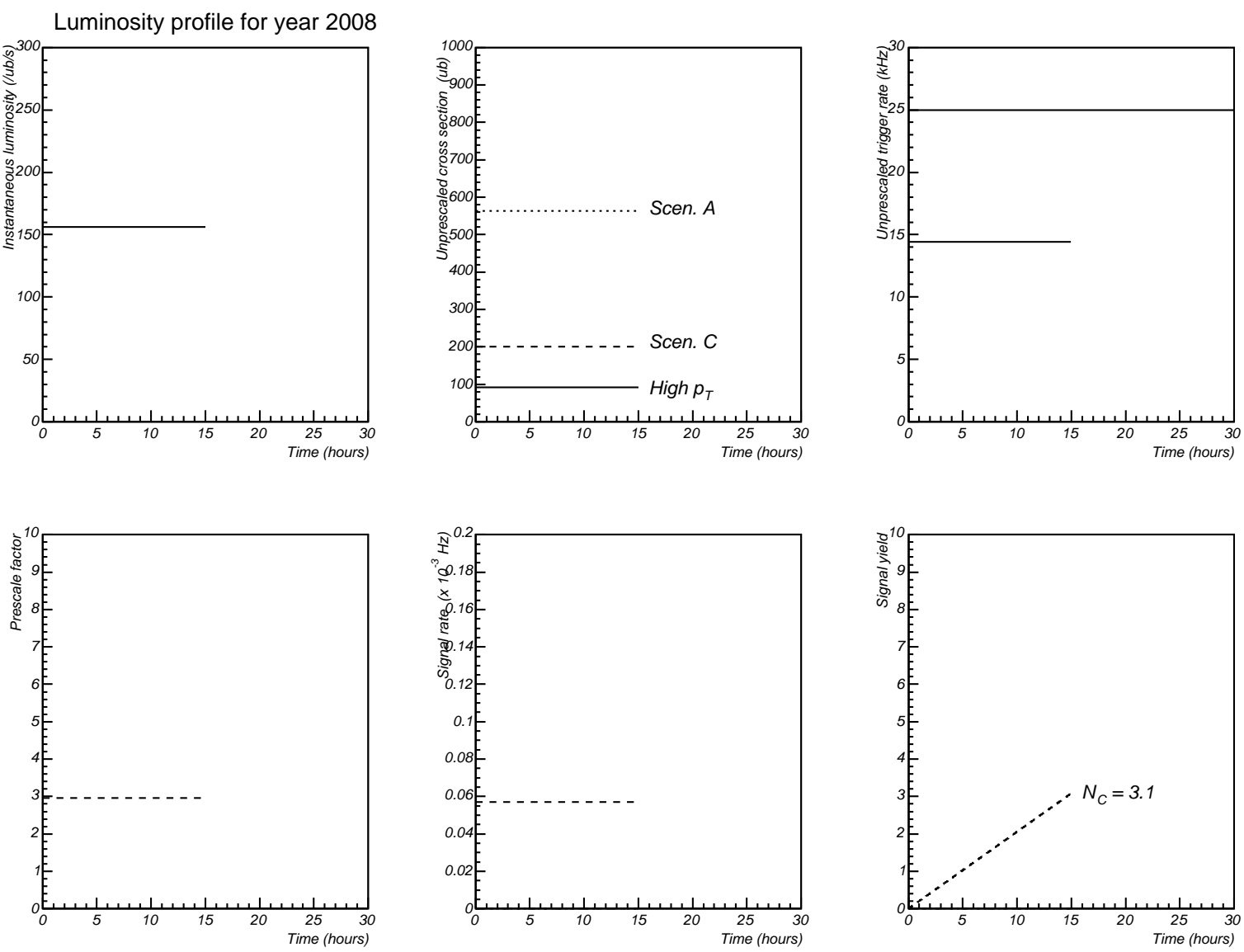

Figure 6: Instantaneous luminosity, cross sections, trigger rates prescales, $B_{s}^{0}$ signal rates and signal yields for a luminosity leveled store in 2008 with the nominal high $p_{T}$ trigger scenario. 


\section{Conclusions}

Table 4 shows that the possible benefits of luminosity leveling depend greatly on the way in which the Tevatron is operated in terms of store duration and initial luminosity, but also on the bandwidth used by the high $p_{T}$ physics program. In the best possible scenario, the maximum benefit of luminosity leveling appears to be slightly less than $30 \%$ but it would be much less if the Tevatron luminosity projections for 2008/9 are met. An actual implementation of luminosity leveling would likely incur a penalty in the total integrated luminosity which might offset any possible gain in the $B_{s}^{0}$ yield found in this study. Based on these observations it appears that great care should be exercised in determining whether to expend the significant effort required to implement luminosity leveling in the Tevatron in the future.

\section{References}

[1] Jeff Spalding, "Cost and Schedule Report/Luminosity Projection", Talk presented at Fermilab DOE mini-review, September 8, 2004.

[2] M. Jones and J.D. Lewis, "A Method for Extrapolating Trigger Rates to High Luminosities", CDF Note 7356, November 8, 2004.

[3] B. J. Kilminster, et al., "XFT Upgrade Options and Studies", CDF Note 7039 v1.4, July 12, 2004.

[4] A. Belloni, et al., "Study of $B_{s}, B^{+}$and $B^{0}$ Yields in the Hadronic B Samples", CDF Note 6614, September 1, 2003. 